\title{
Finite unitary ring with minimal non-nilpotent group of units
}

\author{
Mohsen Amiri, Mostafa Amini \\ Departamento de Matemtica-ICE-UFAM, 69080-900, Manaus-AM, Brazil \\ Department of Mathematics, Payame Noor University, Tehran, Iran
}

\begin{abstract}
Let $R$ be a finite unitary ring such that $R=R_{0}\left[R^{*}\right]$, where $R_{0}$ is the prime ring and $R^{*}$ is not a nilpotent group. We show that if all proper subgroups of $R^{*}$ are nilpotent groups, then the cardinality of $R$ is a power of 2 . In addition, if $(R / J a c(R))^{*}$ is not a $p$-group, then either $R \cong M_{2}(G F(2))$ or $R \cong M_{2}(G F(2)) \oplus A$, where $M_{2}(G F(2))$ is the ring of $2 \times 2$ matrices over the finite field $G F(2)$ and $A$ is a direct sum of copies of the finite field $G F(2)$.
\end{abstract}

2010 MSC :16P10, 16U60, 20D15.

Keywords: Finite ring, Group of units, Nilpotent groups.

\section{Introduction}

The relations between rings and their groups of units are an interesting research subject. In [4], Groza has shown that if $R$ is a finite ring and at most one simple component of the semi-simple quotient ring $R$ by it's Jacobson radical $J a c(R)$ is the field of order 2 , then the group of units $R^{*}$ is a nilpotent group if and only if $R$ is a direct sum of two-sided ideals that are homomorphic images of group algebras of the form $S P$, where $S$ is a particular

E-mail Address: mohsen@ufam.edu.br; mamini1356@yahoo.com 
commutative finite ring, $P$ is a finite $p$-group, and $p$ is a prime number. More recently, Dolzan improved some results of Groza and described the structure of an arbitrary finite ring with a nilpotent group of units, see [1].

Let $X$ be a class of groups. We say that a group $G$ is a minimal non- $X$-group, if $G \notin X$, and all proper subgroups of $G$ belong to $X$. Minimal non- $X$-groups have been studied for various classes of groups $X$. For example, minimal non-abelian groups were studied by Miller and Moreno in [5], while Schmidt in [7] studied minimal non-nilpotent groups and he characterized such finite groups. The natural question is what we can say about a finite ring whose group of units is a minimal non- $X$-group. In this paper, we study finite rings with minimal non-nilpotent groups of units and we prove that the order of such rings is $2^{n}$ for some positive integer $n$. More precisely, we prove the following theorem:

Theorem 1. Let $R$ be a unitary ring of finite cardinality $2^{a} m, m$ be an odd number and $R^{*}$ a minimal non-nilpotent group. Then, $|R|=2^{a}$. Also, if $(R / \operatorname{Jac}(R))^{*}$ is not a p-group, then either $R \cong M_{2}(G F(2))$ or $R \cong M_{2}(G F(2)) \oplus A$, where $A$ is a direct sum of copies of the finite field of order two.

In this paper, from now on $R$ denotes a ring with an identity $1 \neq 0$ and for an arbitrary finite set $X,|X|$ denotes the number of elements in $X$. We denote the group of units of $R$ by $R^{*}$, the order of element $x$ in $R^{*}$ by $o(x)$, and the group generated by $x$ by $\langle x\rangle$. The ring of $n \times n$ matrices over a ring $R$ is denoted by $M_{n}(R)$, and the set of elements that commute with every element of $R$ is denoted by $Z(R)$. The centralizer of the subset $X$ of $R$ is the set of all elements of $R$ which commute with every element of $X$ is denoted by $C_{R}(X)$. Also, for any pair $a, b \in R,[a, b]=a b-b a$ is the Lie product of $a$ and $b$ and $R_{0}[S]$ denotes the subring of $R$ which is generated by $S \subseteq R$ over $R_{0}$, where $R_{0}$ is the prime subring of $R$. The characteristic of $R$ is denoted by $\operatorname{Char} R$ and $G F\left(p^{m}\right)$ is the finite field of order $p^{m}$, where $p$ is a prime number. 


\section{Results}

We begin with the following useful lemma:

Lemma 2. Let $R$ be a unitary finite local ring with a nontrivial minimal ideal $I$ and we assume that $\operatorname{Jac}(R)$ is a commutative ideal. Then $J a c(R) \subseteq \operatorname{ann}_{R}(I)$.

Proof. We have $\operatorname{Jac}(R) I \subseteq I$, and since $I$ is minimal, we deduce that $\operatorname{Jac}(R) I=0$ or $\operatorname{Jac}(R) I=I$. Since $I \neq 0$, by Nakayama's Lemma, we conclude that $\operatorname{Jac}(R) I \neq I$. Therefore $\operatorname{Jac}(R) I=0$.

Remark 1. Let $R=A \oplus B$ be a finite ring where $A$ and $B$ are two ideals in $R$. Then $R^{*}=A^{*} \oplus B^{*}$ and $1=1_{A}+1_{B}$ where $1_{A}$ and $1_{B}$ are the identity elements of $A$ and $B$, respectively. It is clear that $A^{*}+1_{B} \leq R^{*}$ and that $A^{*}+1_{B} \cong A^{*}$.

Lemma 3. Let $R$ be a finite ring. If $|R|$ is an odd number, then $R=R_{0}\left[R^{*}\right]$.

Proof. By Lemma 1.1 from [4], the proof is clear.

Minimal non-nilpotent groups are characterized by Schmidt as follows:

Theorem 4.(see (9.1.1) of [6]) Assume that every maximal subgroup of a finite group $G$ is nilpotent but $G$ itself is not nilpotent. Then:

(i) $G$ is soluble.

(ii) $|G|=p^{m} q^{n}$ where $p$ and $q$ are unequal prime numbers.

(iii) There is a unique Sylow p-subgroup $P$ and a Sylow q-subgroup $Q$ is cyclic. Hence $G=Q P$ and $P \unlhd G$.

Let $R$ be a finite ring with $|R|$ an odd number. Then, in the following theorem, we show that $R^{*}$ is not a minimal non-abelian group. 
Theorem 5. Let $R$ be a finite ring of order $m$ with $m$ an odd number. If every proper subgroup of $R^{*}$ is an abelian group, then $R$ is a commutative ring.

Proof. Consider the finite ring $R$ which is minimal subject to hypothesis of the theorem. Since every maximal subgroup of $R^{*}$ is abelian, $R^{*}$ is a minimal non-abelian group. By Lemma of [2](p.512), every unitary ring of order $p$ or $p^{2}$ for prime number $p$, is a commutative ring. So we may assume that $|R| \notin\left\{p, p^{2}\right\}$. Let $S$ be a proper subring of $R$. From Lemma 3, it follows that $R=R_{0}\left[R^{*}\right]$, and hence $S^{*} \neq R^{*}$. By assumption, $S^{*}$ is an abelian group and by Lemma 3, $S=S_{0}\left[S^{*}\right]$ is a commutative ring. So every proper subring of $R$ is a commutative ring. Let $|R|=p_{1}^{\alpha_{1}} \ldots p_{k}^{\alpha_{k}}$ be the canonical decomposition of $|R|$ to the prime numbers $p_{i}$. Then we know that

$$
R=R_{1} \bigoplus R_{2} \bigoplus \ldots \bigoplus R_{k}
$$

where each ideal $R_{i}$ is of order $p_{i}^{\alpha_{i}}$. Let $H_{i}$ be a subgroup of $R^{*}$ such that $H_{i} \cong R_{i}^{*}$ for all $i$. If $k>1$, then $H_{i}$ is an abelian subgroup of $R^{*}$. By minimality of $R, R_{i}$ is a commutative ring for all $i$ and then $R$ is a commutative ring, which is a contradiction. So, suppose that $|R|=p^{\beta}$, where $p>2$ is a prime number. We have the following two cases with respect to the Jacobson radical, either $\operatorname{Jac}(R)=0$ or $\operatorname{Jac}(R) \neq 0$ :

Case 1. Let $\operatorname{Jac}(R)=0$. By Wedderburn Structure Theorem, $R \cong \bigoplus_{i=1}^{t} M_{n_{i}}\left(D_{i}\right)$ where $D_{i}$ is a finite field. If $t>1$, then by minimality of $R$ and Remark 1, every $M_{n_{i}}\left(D_{i}\right)$ is commutative, and so $R$ is commutative, which is a contradiction. It follows that $t=1$, and so $R \cong M_{n}(D)$, where $D$ is a finite field and $n$ is a positive integer. Since $R$ is not a commutative ring, we have $n>1$. So, $\left(M_{n}(D)\right)^{*} \cong R^{*}$ implies that $R^{*}$ is not a nilpotent group, hence $R^{*}$ is a minimal non-nilpotent group. But any Sylow 2 -subgroup of $R^{*}$ is neither cyclic nor normal, which is a contradiction by Theorem 4(iii).

Case 2. Let $\operatorname{Jac}(R) \neq 0$. First suppose that $R^{*}$ is a nilpotent group. Since $-1 \in R^{*}$ and $o(-1)=2$, we deduce that 2||$R^{*} \mid$. Since $\operatorname{Jac}(R) \neq 0$, by Lemma 1.2 of [4], $1+\operatorname{Jac}(R)$ is a $p$-group. Let $P \in S y l_{p}\left(R^{*}\right)$ and $K$ be a subgroup of $R^{*}$ such that $R^{*}=P K$ and $P \cap K=1$. By assumption, $P$ and $K$ are abelian groups, and hence $R^{*}$ is an abelian group. Thus by Lemma 3, $R=R_{0}\left[R^{*}\right]$ is commutative, which is a contradiction. It follows that $R^{*}$ is not a nilpotent group, and it follows that $R^{*}$ is a minimal non-nilpotent group. 
By Theorem 4, $\left|R^{*}\right|=r^{m} q^{n}$ where $r, q$ are prime numbers. Also, since 2||$R^{*} \mid$ and by Lemma (1.2) of [4], $1+\operatorname{Jac}(R)$ is a $p$-group, we may assume that $r=p$ and $q=2$. On the other hand, $(R / J a c(R))^{*}=R^{*}+\operatorname{Jac}(R) / \operatorname{Jac}(R)$, and so every proper subgroup of $(R / J a c(R))^{*}$ is an abelian group. By minimality of $R, R / J a c(R)$ is a commutative ring and hence, we deduce that $[R, R] \subseteq \operatorname{Jac}(R)$. Let $P \in \operatorname{Syl}_{p}\left(R^{*}\right)$ and $Q \in \operatorname{Syl}_{2}\left(R^{*}\right)$. By Theorem 4(iii), either $P \triangleleft R^{*}$ or $Q \triangleleft R^{*}$. We claim that $P \triangleleft R^{*}$. Otherwise, by Theorem 4(iii), $P=\langle z\rangle$ is a cyclic subgroup of $R^{*}$, where $z \in P$. Since $1+\operatorname{Jac}(R) \leq P$, there is a positive integer $i$ such that $H=1+\operatorname{Jac}(R)=\left\langle z^{i}\right\rangle$. Since $H \triangleleft R^{*}$ and $R^{*}$ is a nonnilpotent group, $H \neq P$. Since $H Q$ is an abelian subgroup of $R^{*}$ and $R=\langle z, Q\rangle$, we have $H \leq Z\left(R^{*}\right)$, and so $H \leq Z(R)$. Consequently, $\operatorname{Jac}(R) \subseteq Z(R)$. Since $[R, R] \subseteq J a c(R)$, we have $u v-v u \in J a c(R)$ for all $u, v \in R^{*}$. Thus, it follows that $u v u^{-1} v^{-1}-1 \in \operatorname{Jac}(R) \subseteq$ $Z(R)$, and so $u v u^{-1} v^{-1} \in Z(R)$ for all $u, v \in R^{*}$. Hence $R^{*}$ is a nilpotent group, which is a contradiction. Therefore $P \triangleleft R^{*}$, as claimed. By Theorem 4 (iii), $Q=\langle x\rangle$ for some $x \in Q$. Now, we claim that $R$ is a local ring. Let $\left\{M_{1}, \ldots, M_{k}\right\}$ be the set of all maximal ideals of $R$, where $k>1$. Since $R / \operatorname{Jac}(R)=R /\left(M_{1} \cap \ldots \cap M_{k}\right) \cong R / M_{1} \times \ldots \times R / M_{k}$, we conclude that $(R / J a c(R))^{*} \cong\left(R / M_{1}\right)^{*} \times \ldots \times\left(R / M_{k}\right)^{*}$. Let $\bar{Q} \in \operatorname{Syl}_{2}\left(\left(R / M_{1} \times \ldots \times R / M_{k}\right)^{*}\right)$. Then, since 2||$\left(R / M_{1}\right)^{*} \mid$ for all $1 \leq i \leq k$, we see that $\bar{Q}$ is not a cyclic group. Also, we have $(R / J a c(R))^{*}=R^{*}+\operatorname{Jac}(R) / J a c(R)=P Q+\operatorname{Jac}(R) / J a c(R)$, and so $Q$ is not a cyclic group, which is a contradiction. Hence $k=1$, as claimed. Let $M=\operatorname{Jac}(R)$. Since $1+M$ is an abelian group, $M$ is a commutative ideal. Since $R / M$ is a finite field, $M$ is not a central ideal. So, there exists $w \in M$ such that $w x \neq x w$. By minimality of $R$, we have $R=R_{0}[w, x]$. Let $I$ be a minimal ideal of $R$. We follow the proof by separating two subcases, either $Z(R) \cap I \neq 0$ or $Z(R) \cap I=0$ :

Subcase 1. Let $0 \neq a \in Z(R) \cap I$. By Lemma 2, $M \subseteq a n_{R}(I)=\{r \in R: r s=$ 0 for all $s \in I\}$. Since $R / M$ is a finite field, $M=a n_{R}(I)$. It follows from $a \in Z(R)$ that $I=R a$ is two sided ideal. Since $R / M$ is a finite field, $(R / M)^{*}=\langle x+M\rangle$ for some $x \in R$ with $\operatorname{gcd}(o(x+M), p)=1$. Let $y \in R \backslash M$. Therefore $y+M=x^{i}+M$ for some integer $0 \leq i \leq n-1$. Then $y=x^{i}+s$ for some $s \in M$ and this, implies that $y a=x^{i} a+s a=x^{i} a$. So, we have $I=\left\{0, x a, \ldots, x^{n} a\right\} \subseteq M$. Since $x x^{i} a=x^{i} a x, w\left(x^{i} a\right)=\left(x^{i} a\right) w$ and $R=$ $R_{0}[x, w]$, we conclude that $x^{i} a \in Z(R)$, and so $I \subseteq Z(R)$. The minimality of $R$ implies that $R / I$ is a commutative ring, and consequently $[R, R] \subseteq I \subseteq Z(R)$. Thus, we have 
$u v u^{-1} v^{-1}-1 \in I \subseteq Z(R)$ for all $u, v \in R^{*}$. It follows that $u v u^{-1} v^{-1} \in Z\left(R^{*}\right)$, and hence $R^{*}$ is a nilpotent group, which is a contradiction.

Subcase 2. Let $Z(R) \cap I=0$ and $0 \neq b \in I$. Since $R=R_{0}[w, x]$ and $M$ is commutative, we have $b w=w b$, and so $[b, x] \neq 0$. Therefore $R=R_{0}[b, x]$. We may assume that $b=w \in I$ and $m_{1}, m_{2} \in M$. Since $M$ is a commutative ring and $x m_{1}, m_{2} x \in M$, we have

$$
\left(x m_{1}\right) m_{2}=m_{2}\left(x m_{1}\right)=\left(m_{2} x\right) m_{1}=m_{1} m_{2} x .
$$

Since $R=R_{0}[x, w]$ and $w m_{1} m_{2}=m_{1} m_{2} w$, we conclude that $M^{2} \subseteq Z(R)$. If $M^{2} \neq 0$, then by minimality of $R, R / M^{2}$ is a commutative ring, and so $0 \neq[R, R] \subseteq M^{2} \cap I$. Since $I$ is a minimal ideal and $M^{2}$ is an ideal, $I \subseteq M^{2} \subseteq Z(R)$, which is a contradiction. Hence $M^{2}=0$, and so by considering $R$ as a local ring, for all $s \in M \backslash\{0\}$, we have $M \subseteq \operatorname{ann}_{R}(s)$. We claim that $I=M$, otherwise consider $l \in M \backslash I$. Since $R=R_{0}[x, w]$, we have $l=\sum n_{i} x^{i}+c$, where $c \in I$ and $n_{i} \in R_{0}$. Therefore $l-c=\sum n_{i} x^{i} \in M$. Then $\sum n_{i} x^{i} \in Z(R)$. If $l-c \neq 0$, then by minimality of $R$, we have that $R / R(l-c)$ is a commutative ring. By a similar argument to the one subcase 1 , we have $R(l-c) \subseteq Z(R)$. From $0 \neq[R, R] \subseteq R(l-c) \cap I$, where $I$ is a minimal ideal, follows that $I \subseteq R(l-c) \subseteq Z(R)$, which is a contradiction. Then $l-c=0$, which is a contradiction. Therefore $M=I$. Since $R / M$ is a finite field, we have $R=R^{*} \cup M$. Then $\left|R^{*}\right|=|R|-|M|=o(x)|P|$. Let $|R / M|=p^{m}$. Then $|R|=p^{m}|M|$, and so $|R|-|M|=\left(p^{m}-1\right)|M|$, consequently, $1+M=P$. Since $P\left\langle x^{2}\right\rangle$ is an abelian subgroup of $R^{*}$ and $R=R_{0}[w, x]$, we conclude that $x^{2} \in Z(R)$. If $p \mid o(x+h)$ for some $h \in P$, then $e=(x+h)^{\frac{o(x+h)}{p}} \in P=1+M$, and so $e-1 \in M$. Since $R=R_{0}[x+h, w]$ and $M$ is commutative, $e-1 \in Z(R)$. Therefore $M \cap Z(R) \neq 0$, which is a contradiction. It follows that $\operatorname{gcd}(o(x+h), p)=1$ for all $h \in M$. By a similar argument to the one above, $(x+h)^{2} \in Z(R)$ for all $h \in M$. Then $x^{2}+x h+h x+h^{2} \in Z(R)$. Since $h^{2}=0$ and $x^{2} \in Z(R)$, we have $x h+h x \in Z(R) \cap M=0$. Therefore $x h=-h x$ for all $h \in M$. Let $0 \neq h \in M$. Since $(R / M)^{*}=\langle x+M\rangle$, we deduce that $x+1+M=x^{t}+M$ for some integer $t$, and then $q=x^{t}-x-1 \in M$. Since $M$ is a commutative ideal, $q w=w q$. It follows from $q x=x q$ and from $R=R_{0}[x, w]$ that $q=x^{t}-x-1 \in M \cap Z(R)=0$. So, $x+1=x^{t}$. Since $x+1=x^{t} \notin Z(R)$, we deduce that $t$ is an odd number. Then, we have $x^{t} h=(-1)^{t} h x^{t}=-h x^{t}$. Therefore, $(x+1) h=-h(x+1)$. But $(x+1) h=x h+h=-h x+h=-h(x+1)$. Hence $2 h=0$, 
and so $h=0$ for all $h \in M$, which is a contradiction.

Theorem 6. Let $R$ be a finite ring of order $p^{m}$ where $p$ is an odd prime number. If every proper subgroup of $R^{*}$ is nilpotent, then $R$ is a commutative ring.

Proof. Consider the finite ring $R$ which is minimal with respect to these conditions, but it is not commutative. Then $R^{*}$ is a minimal non-nilpotent group. By a similar argument to the one in case 1 of the previous theorem, we may assume that $\operatorname{Jac}(R) \neq 0$. By Theorem 4, $\left|R^{*}\right|=r^{m} q^{n}$ where $r$ and $q$ are prime numbers. By Lemma 1.2 from [4], $1+\operatorname{Jac}(R)$ is a $p$-group and then $r=p$. Since $-1 \in R^{*}$ and $o(-1)=2$, we have $q=2$. Let $P \in \operatorname{Syl}_{p}\left(R^{*}\right)$ and $Q \in \operatorname{Syl}_{2}\left(R^{*}\right)$. Let $I$ be a minimal ideal of $R$ that is contained in $\operatorname{Jac}(R)$. Then $I^{2}=0$, and hence $I$ is commutative. By minimality of $R$, we have that $R / I$ is commutative, so $[R, R] \subseteq I$. We have two cases with respect to $I \cap Z(R)$, either $I \cap Z(R) \neq 0$ or $I \cap Z(R)=0$

Case 1. Suppose that $I \cap Z(R) \neq 0$. If $0 \neq c \in I \cap Z(R)$, then by a similar argument to the one in the subcase 1 of case 2 , in the above theorem, $I \subseteq Z(R)$, and then $1+I \leq Z\left(R^{*}\right)$. Since $u v u^{-1} v^{-1}-1 \in I$, we have $\left(R^{*}\right)^{\prime} \leq Z\left(R^{*}\right)$, and so $R^{*}$ is a nilpotent group, which is a contradiction.

Case 2. Let $I \cap Z(R)=0$. First suppose that $1+I \neq P$. Since $1+I \triangleleft P$, there exists $c \in I \backslash\{0\}$ such that $1+c \in Z(P)$. Also, $(1+I) Q$ is a proper nilpotent subgroup of $R^{*}$. So $1+I \leq C_{R^{*}}(Q)$, and hence $c \in Z(R)$, which is a contradiction. Therefore $1+I=P$ is an abelian subgroup of $R^{*}$. By Theorem 4 , either $P$ is cyclic or $Q$ is cyclic. Since $1+I=P \triangleleft R^{*}$, by Theorem 4, $Q$ is cyclic. Since $P$ is an abelian group and $Q$ is cyclic, every proper subgroup of $R^{*}$ is an abelian group, which is a contradiction by Theorem 5 .

If 2||$R \mid$, then the above theorem is no longer valid. For example, let $R$ be the set of all $2 \times 2$ matrices over the finite field $G F(2)$. Then $R^{*} \cong S_{3}$, where $S_{3}$ is the symmetric group of order 6 and clearly, $S_{3}$ is a minimal non-abelian group. For simplicity, let $\Delta$ be the set of all rings $R$ in which either $R \cong M_{2}(G F(2))$ or $R \cong M_{2}(G F(2)) \oplus A$, where $A$ is a direct sum of copies of the finite field of order two. 
Remark 2. Let $S l\left(2, G F\left(2^{m}\right)\right)$ be the kernel of the homomorphism $\operatorname{det}\left(M_{n}\left(G F\left(2^{m}\right)\right) \longrightarrow\right.$ $G F\left(2^{m}\right)^{*}$. We recall that when $m>1$, then $S l\left(2, G F\left(2^{m}\right)\right)^{*}=\left(\left(S l\left(2, G F\left(2^{m}\right)\right)\right)^{*}\right)^{\prime}$, and hence for $n>1$ and $m>1$, we have that $\left(M_{n}(D)\right)^{*}$ is not a minimal non-nilpotent group.

Theorem 7. Let $R$ be a unitary non-commutative ring of finite cardinality $2^{\beta}$ such that every proper subgroup of $R^{*}$ is a nilpotent group.

(a) If $\operatorname{Jac}(R)=0$, then $R \in \Delta$.

(b) If $\operatorname{Jac}(R) \neq 0$, then $(R / \operatorname{Jac}(R))^{*}$ is a cyclic $p$-group for some odd prime number $p$.

Proof. (a) We proceed by induction on $\beta$. Since $R$ is a simple artinian ring, by the structure theorem of Artin-Wedderburn, we have $R \cong \bigoplus_{i=1}^{t} M_{n_{i}}\left(D_{i}\right)$, where every $D_{i}$ is a finite field. If $t=1$, then $R \cong M_{n_{1}}(D)$, and clearly, $n_{1}=2$ and $D \cong G F(2)$, so $R \in \Delta$. Let $t>1$ and $n_{i}>1$ for some $1 \leq i \leq t$. By Remark 2, if $M_{n_{i}}\left(D_{i}\right)$ is a minimal nonabelian group, then, $n_{i}=2$ and $D_{i}=G F(2)$. If for some $j \neq i$, we have $n_{j}>1$, then $R^{*}$ is not a minimal non-abelian group, which is a contradiction. Therefore $M_{n_{j}}\left(D_{j}\right) \cong D_{j}$ for all $j \neq i$. Let $H \leq R^{*}$ such that $H \cong\left(M_{n_{i}}(G F(2))\right)^{*}$. If $\left|D_{j}^{*}\right|>1$ for some $j \neq i$, then $R^{*} \neq H$, and hence $H$ is a non-nilpotent proper subgroup of $R^{*}$, which is a contradiction. Consequently, $D_{j} \cong G F(2)$, and hence $R \in \Delta$.

(b) Suppose for a contradiction that $(R / \operatorname{Jac}(R))^{*}$ is not a $p$-group. We may assume that $|R|$ is minimal such that $(R / \operatorname{Jac}(R))^{*}$ is not a $p$-group. By Theorem $4, R^{*}=P Q$, where $P \triangleleft G$ and $Q$ is a cyclic Sylow subgroup. Let $I \subseteq \operatorname{Jac}(R)$ be a minimal ideal of $R$. It is easy to see that $\operatorname{char}(I)=2$ and $I^{2}=0$. Therefore, $1+I$ is an elementary abelian 2-group. Also, $(R / I) /(\operatorname{Jac}(R / I)) \cong R / \operatorname{Jac}(R)$ implies that $((R / I) /(\operatorname{Jac}(R / I)))^{*}$ is not a $p$-group, so by minimality of $R,(R / I)^{*}$ is a nilpotent group. Let $p>2$ be a prime number such that $p|| R^{*} \mid$. Clearly, $2 p||(R / \operatorname{Jac}(R))^{*}$. Let $\left\{M_{1}, \ldots, M_{k}\right\}$ be the set of all maximal ideals of $R$. Then, we have

$$
R / J a c(R) \cong R / M_{1} \oplus \ldots \oplus R / M_{k}
$$

Since $R / M_{i}$ is a simple ring, $R / M_{i} \cong M_{n_{i}}\left(G F\left(2^{m_{i}}\right)\right)$ for some positive integers $n_{i}$ and $m_{i}$. If $n_{i}>1$ for some $\mathrm{i}$, then $R / M_{i} \cong M_{n_{i}}\left(G F\left(2^{m_{i}}\right)\right)$. Let $x$ and $y$ be two arbitrary 
elements of $R^{*}$ such that $x y \neq y x$ and $\operatorname{gcd}(o(x), o(y))=1$. Since $(R / I)^{*}$ is a nilpotent group, we have $x y-y x \in I \subseteq \operatorname{Jac}(R)$. Hence $n_{i}=1$ for all $i$, and so $R / M_{i}$ is a finite field. But $\operatorname{gcd}\left(\left|\left(R / M_{i}\right)^{*}\right|, 2\right)=\operatorname{gcd}\left(2^{m_{i}}-1,2\right)=1$ for all $i$, so $2 \nmid \mid(R / \operatorname{Jac}(R))^{*}$, which is a contradiction.

Here we give an example for the statement of Theorem 7 part (b). Let $G F(2)[x, y]$ be the free ring generated with two elements $x$ and $y$ over finite field $G F(2)$. Let $H$ be the ideal generated by $\left\{x^{2}, y^{3}+y+1, x y-y^{2} x\right\}$. Let $R=G F(2)[x, y] / H$, and let $I$ be the ideal generated with $x+H$ in $R$. Since $x y-y x \notin H, R$ is a non-commutative ring. Let $L$ be the ideal generated by $t^{3}+t+1$ in $Z_{2}[t]$. Since $R / I \cong Z_{2}[t] / L$ and $L$ is a maximal ideal, $R / I$ is a finite field of order 8 . Let $(R / I)^{*}=\langle u+I\rangle$. It is easy to check that $I=\left\{0, x+H, u x+H, \ldots, u^{7} x+H\right\}$. Clearly, $1+I$ is an elementary abelian 2-group and $R^{*}=(1+I)\langle u\rangle$ is a minimal non-nilpotent group.

Now we are ready to prove Theorem 1 .

Proof. By Theorem $\left[6, \alpha_{1} \geq 1\right.$. Let $|R|=2^{\alpha_{1}} p_{2}^{\alpha_{2}} \ldots p_{k}^{\alpha_{k}}$ be the canonical decomposition of $|R|$ to the prime numbers $p_{i}$. Then

$$
R=R_{1} \oplus R_{2} \oplus \cdots \oplus R_{k}
$$

where each ideal $R_{i}$ is of order $p_{i}^{\alpha_{i}}$. By Theorem $6, R_{2} \oplus \cdots \oplus R_{k}$ is a commutative ring, and hence $\left(R_{1}\right)^{*}$ is a minimal non-nilpotent group. Consequently, $k=1$. The rest of proof is clear by Theorem 7 .

\section{References}

[1] D. Dolzan, Nilpotency of the group of units of a finite ring, Bull. Aust. Math. Soc. 79 (2009) 177-182.

[2] K. E Eldridge, Orders for finite noncommutative rings with unity, The American Mathematical Monthly, 75, 5, (1968) 512-514. 
[3] B. Farb, R. Keith Dennis, Noncommutative algebra, Graduate texts in mathematics, Springer Verlag, New York, 144, (1993).

[4] G. Groza, Artinian rings having a nilpotent group of units, Journal of Algebra, 121 (1989) 253-262.

[5] G. A. Miller and H. C. Moreno. Nonabelian groups in which every subgroup is abelian, Trans. Amer. Math. Soc,4, no. 4 (1903) 398-404.

[6] D.J.S. Robinson, A course in the theory of groups, Springer Verlag, New York, (1980).

[7] O. J. Schmidt. ber Gruppen, deren smtliche Teiler spezielle Gruppen sind. Mat. Sbornik, 31, (1924) 366-372.

[8] J. H. M. Wedderburn, A theorem on finite algebra, Trans. Amer. Math. Soc. 6 (1905) 349-352. 